\title{
Les substances végétales non volatiles et leur effet phagostimulant sur les larves de cinquième stade de la teigne du poireau, Acrolepiopsis assectella (Lep.)
}

\author{
H. Al-Rouz et E. Thibout
}

Institut de biocénotique expérimentale des agrosystèmes (UA CNRS 340), Parc de Grandmont, avenue Monge, 37200 Tours

(reçu le 21-7-1988, accepté le 14-12-1988)

Résúmé - La phagostimulation des larves de $5^{\theta}$ stade de la teigne du poireau est étudiée sur un support en polystyrène à l'aide de composés non volatiles déjà identifiés dans les Allium. Les cires épicuticulaires, la sinigrine, certains acides aminés dont la méthionine et la majorité des flavonoïdes testés ne sont pas stimulants. Les substances phagostimulantes se répartissent en 2 groupes : d'une part, les substances spécifiques des Allium tels les alkyl-cystéine-sulfoxides et la cystéine qui peut être rangée dans ce groupe; d'autre part, des composés plus généraux comme certains hexoses, le xylose, plusieurs stéroïdes et saponines, un flavonoïde et le chlorure de sodium. L'acide chlorogénique est, en revanche, antiappétent. Un effet propre ou synergique de plusieurs composés actifs est recherché.

phagostimulation larvaire - composés soufrés - substances générales — plante-hôte Allium - cires

Summary - Non volatile plant chemicals and phagostimulation in the fifth instar larvae of the leek-moth, Acrolepiopsls assectella (Lep.). Phagostimulation was studied on polystyrene substrate in the leek-moth larvae with compounds already identified in Allium plants. Epicuticular waxes, sinigrin, some amino-acids among which methionine and the majority of tested flavonoids are not phagostimulant. There are 2 groups of phagostimulant compounds. The first includes specific compounds of Allium like S-alkyl-cystein-sulfoxides and also cysteine which can be placed in this group. The other includes more general compounds like carbohydrates (some hexoses and the xylose), several steroids and saponins, one flavonoid and sodium chloride. On the other hand, chlorogenic acid is a phagorepellent. An intrinsic or synergistic effect of these active compounds is analysed in relation with their presence and abundance in the host plant. larval phagostimulation - sulfur compounds - general substances - host-plant - Allium
- waxes

\section{Introduction}

Des signaux émis par les plantes conditionnent en partie la prise de nourriture des insectes phytophages. Cette phagostimulation a été étudiée antérieurement chez les larves de $5^{\mathrm{e}}$ stade (L5) de la teigne du poireau, Acrolepiopsis assec- 
tella Zeller (Hyponomeutoïdea), à l'aide d'un support en polystyrène et d'extraits méthanoliques d'une trentaine de végétaux (Al-Rouz et Thibout, 1988a). La prise de nourriture de cet insecte, dont la larve mine l'intérieur des feuilles des plantes du genre Allium, pourrait être stimulée par 2 types de substances d'origine végétale, certaines trouvées chez un grand nombre de plantes, d'autres plus spécifiques, particulières au genre Allium.

Alors que les substances allélochimiques volatiles responsables de l'attraction des adultes et des larves d'A. assectella sont assez bien connues (Lecomte et Thibout, 1981; Al-Rouz et Thibout, 1988b), il n'en est pas de même des substances agissant par gustation. Les substances permettant la sélection alimentaire par les larves, et plus particulièrement celles intervenant dans la prise de nourriture, ont été recherchées. Bien que cette sélection puisse avoir lieu à l'un quelconque des stades larvaires, les L5 ont été seules étudiées, étant plus faciles à manipuler.

Cette première étude de la phagostimulation chez $A$. assectella a été réalisée essentiellement à l'aide de substances non volatiles déjà identifiées dans les Allium.

\section{Matériel et Méthodes}

Les expériences ont été réalisées sur les $\mathrm{L} 5$ issues d'un élevage renouvelé annuellement. La ponte et le développement larvaire s'effectuaient sur la plante-hôte, le poireau Allium porrum (Liliaceee) cultivar Malabare, dans les conditions suivantes : photophase de $18 \mathrm{~h}$ à $26{ }^{\circ} \mathrm{C}$ et $60 \pm 5 \%$ d'humidité relative, scotophase de $6 \mathrm{~h}$ à $18{ }^{\circ} \mathrm{C}$ et $80 \pm 5 \%$ d'humidité relative.

Afin d'éviter toute interférence olfactive, les expériences ont eu lieu dans une pièce n'ayant jamais renfermé de poireau. Les conditions y étaient les suivantes : $12 \mathrm{~h}$ de photophase par nycthémère, $26^{\circ} \mathrm{C}$ constants et $70 \pm 5 \%$ d'humidité relative. Comme décrit antérieurement (Al-Rouz et Thibout, 1988a), le pouvoir phagostimulant des substances végétales a été testé à l'aide de supports artificiels constitués de morceaux de polystyrène de $22 \times 10 \times 2 \mathrm{~mm}$ (Meisner et Asher, 1968). Dix L5, à jeun depuis $2 \mathrm{~h}$, ont été placées dans l'après-midi au centre d'une boîte de Pétri, à égale distance de 2 supports, l'un imprégné du ou des produits à étudier, l'autre servant de témoin. Une vingtaine de boîtes ont été utilisées à chaque test. Leur couvercle était percé en son centre afin qu'un morceau de coton humide y maintienne une forte humidité relative. Le support témoin, suite aux études antérieures (Al-Rouz et Thibout, 1988a), était badigeonné uniformément d'une solution de saccharose à $0,1 \%\left(3.10^{-4}\right.$ $\mathrm{M} / \mathrm{l})$. Cela correspondait à $4,3 \pm 0,2 \cdot 10^{-2} \mathrm{mg}$ de saccharose en moyenne par support. Le support essai a reçu de la même façon la solution de saccharose, puis, après séchage, une solution plus ou moins concentrée de la substance à analyser. Les supports ont été pesés avant d'être badigeonnés, puis pesés à nouveau $24 \mathrm{~h}$ après avoir reçu les diverses substances. $\mathrm{Ce}$ laps de temps est nécessaire afin de permettre l'évaporation complète des solvants. Enfin, ils étaient pesés une dernière fois $24 \mathrm{~h}$ après la fin de l'expérience qui dure elle-même $24 \mathrm{~h}$. La précision des mesures, due à la sensibilité de la balance, était de $2,5 \mu \mathrm{g}$.

Les différences de poids avant et après expérience ont permis d'apprécier les quantités de support consommées. Le pouvoir phagostimulant d'une substance a été évalué par le rapport $R, R$ étant la quantité moyenne de support essai consommé divisée par la quantité totale moyenne consommée sur les 2 supports et variant de 0 à 1 . Si $R=0,5$, les 2 supports sont consommés de façon égale. Si $0<R<0,5$, le support témoin est le plus consommé, la substance est antiappétente. Si $0,5<R<1$, le support essai est le plus consommé, la substance est phagostimulante. Le test du rang et du signe par paires de Wilcoxon a permis de savoir si les poids consommés sur les 2 supports étaient égaux et donc si $R$ était ou non significativement différent de 0,5 .

$R$ a été utilisé car il permet de diminuer l'influence de la variation des témoins dans l'expression des résultats. En effet, les nombreuses expériences ont été échelonnées sur plusieurs mois et intéressaient plusieurs générations. D'autre part, la qualité du poireau et donc l'alimentation larvaire avant l'expérience 
variaient au cours de l'année. Cependant, des analyses de variances effectuées sur le poids des témoins à un moment donné ont montré que ceux-ci étaient homogènes, à l'exception de l'expérience avec le PCSO. Dans ce cas, en augmentant la concentration de PCSO, on augmentait considérablement le pouvoir phagostimulant du support essai et le poids du support témoin consommé diminuait proportionnellement.

Les substances non volatiles testées se répartissent en 2 groupes:

\section{- substances spécifiques des Allium}

- cires épicuticulaires de poireau obtenues par cristallisation à $-18^{\circ} \mathrm{C}$ d'un extrait superficiel produit par immersion des feuilles pendant 30 sec dans le dichlorométhane;

- propyl cystéine sulfoxide (PCSO), un des précurseurs des subtances volatiles soufrées. Trois concentrations ont été testées;

- aginoside, saponine extraite du poireau.

- substances non spécifiques

- sucres : saccharose, fructose, glucose galactose et xylose. Le saccharose a été testé à plusieurs concentrations et les autres sucres à $6.10^{-4} \mathrm{M} / \mathrm{(}(10 \%)$;

- acides aminés : cystéine, méthionine, alanine et valine;

- stérö̈des : cholestérol, $\beta$-sitostérol, $\alpha$ - et B-amyrine, diosgénine (sapogénine);

- flavonoïdes : quercétine, morine, myricétine et acide chlorogénique;

- acide ascorbique (vitamine C);

- chlorure de sodium, inclus ici pour comparaison avec d'autres espèces d'insectes;

- sinigrine, substance soufrée spécifique des crucifères, précurseur de l'isothiocyanate d'allyle volatil.

Les substances insolubles dans l'eau ont été utilisées en solution alcoolique et certains stéroïdes en solution dans l'hexane. D'autre part, les cires épicuticulaires n'étant solubles que dans des solvants attaquant le polystyrène, celles-ci ont été testées sur du papier-filtre. Dans ce cas, supports tests et témoins sont en papier et les pesées étaient alors effectuées après passage $24 \mathrm{~h}$ dans un dessiccateur. Enfin, pour les expériences avec les sucres, le saccharose a été testé avec un support témoin dépourvu de toute substance. Les autres sucres ont été déposés eux aussi isolément sur le support essai, mais le témoin était recouvert de saccharose, comme dans les expériences avec les autres composés.

Enfin, il a été procédé sur les $L 5$ d'A. assectella à une série d'expériences sans saccharose, afin de préciser chez quelques com- posés la présence ou l'absence d'action synergique avec ce sucre. La méthode et les conditions expérimentales étaient identiques à celles utilisées auparavant, mais le support témoin était recouvert uniquement de solvant tandis que le support essai n'était recouvert que de l'une des solutions à étudier. Celles-ci, moins nombreuses, étaient aux mêmes concentrations que lors des expériences précédentes.

\section{Résultats}

Même à la plus faible concentration, le support avec saccharose est plus consommé que le témoin polystyrène seul et la quantité consommée par les L5 augmente avec la quantité de sucre déposée, $R$ étant toujours proche de 1 (Fig. 1). Les

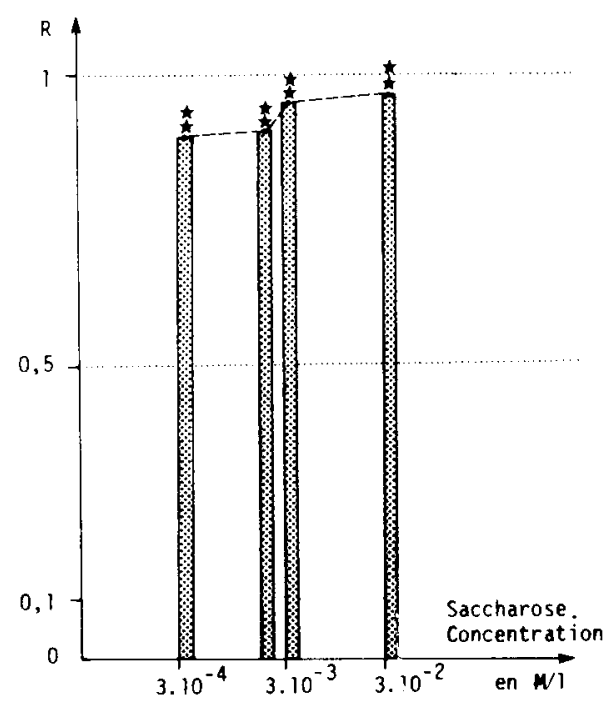

Fig. 1. Rapports $\mathrm{R}$ des poids moyens consommés par les $L 5$ d'A. assectella en présence de diverses concentrations de saccharose. $R=$ $E / E+T, E$ étant le poids moyen de support essai consommé et $T$ le poids moyen de support témoin consommé. Seul le support essai est badigeonné de saccharose. La présence d'astérisque indique que $E$ et $T$ sont significativement differents, soit $R \neq 0,5$ (test de Wilcoxon). ${ }^{*}: 0,05>P>0,01 ;{ }^{* *}: 0,01>P$. 
autres hexoses, comparés au saccharose, sont tout aussi phagostimulants que ce dernier puisque les $L 5$ consomment de façon comparable les 2 supports, $R$ ne différant pas significativement de 0,5 (Fig. 2). Quant au xylose, il est plus phagostimulant que le saccharose; la différence de quantité consommée est significative $(P<0,01)$.

Avec le PCSO, ici encore, la quantité de support consommé s'accroît avec la concentration (Fig. 3). A $10^{-4} \mathrm{M} / \mathrm{l}$, le PCSO n'est pas stimulant mais le devient à $10^{-2} \mathrm{M} /$.

Avec l'aginoside, la prise de nourriture est significativement supérieure à celle du

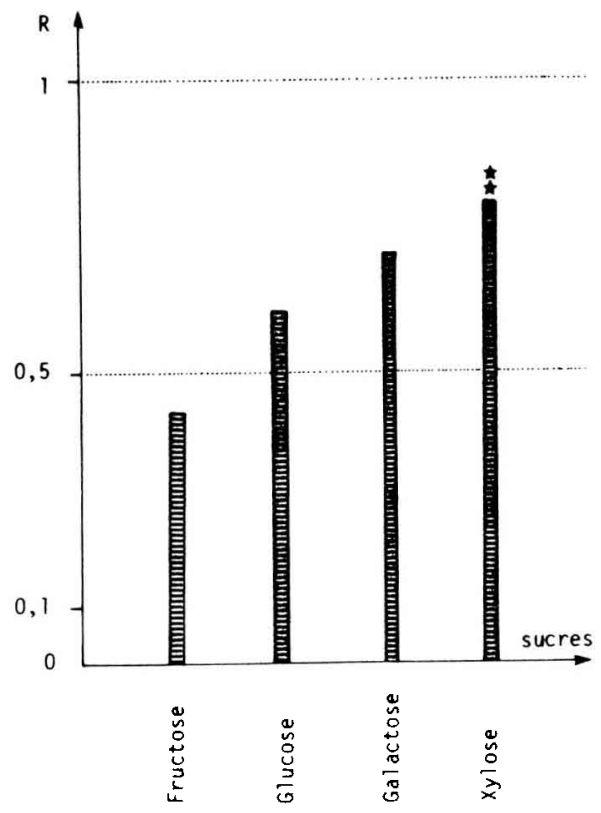

Fig. 2. Rapports $\mathrm{R}$ des poids moyens consommés par les L5 d'A. assectella en présence de différents sucres testés à la concentration de $6.10-4 \mathrm{M} /$. Seul le support témoin est badigeonné de saccharose à $3 \cdot 10^{-4} \mathrm{M} /$. La présence d'astérisque indique que $E$ et $T$ sont significativement différents, soit $R \neq 0,5$ (test de Wilcoxon). ${ }^{*}: 0,05>P>0,01 ;{ }^{* *}: 0,01>P$. témoin (Fig. 4). Inversement, les cires épicuticulaires ne provoquent aucun accroissement significatif de la prise de nourriture par les $L 5(R=0,54)$. II faut noter que c'est lors de ce test que le support témoin a été le plus consommé $\left(40,6.10^{-2} \mathrm{mg}\right.$ de papier, contre $9,7.10^{-2} \mathrm{mg}$ au maximum avec support polystyrène).

Quatre acides aminés ont été proposés aux L5: 2 soufrés, la cystéine ef la méthionine, et 2 non soufrés, proches, la valine et l'alanine. Seule de ces substances, la cystéine a une action significative sur la prise de nourriture (Fig. 4).

A la concentration testée, la vitamine $C$ a un effet phagostimulant tandis que la sinigrine des crucifères est inactive (Fig. 4). II est d'autre part intéressant de constater que tous les stéroïdes ici utilisés entraînent une forte consommation du support sur lequel ils se trouvent ( $R$ proche de 1). Quant aux 4 flavonoïdes testés, 2 se montrent phagostimulants (Fig. 4), mais la quercétine qui est la seule des 4 à être présente dans les Allium (Starke et Hermann, 1976) est particulièrement active.

Enfin, le chlorure de sodium est légèrement phagostimulant à la concentration testée (Fig. 4) puisque $R$ est égal à 0,64 , significativement différent de $0,5 \quad(P<$ 0,05).

Des expériences sans saccharose ont précisé la synergie entre quelques composés et ce sucre (Fig. 5). Dans presque tous les cas, la consommation du support essai est assez semblable à celle obtenue en présence de saccharose. Même sans saccharose, $\mathrm{R}$ est toujours supérieur à 0,5 , et à l'exception de la cystéine, les substances ici testées restent fortement phagostimulantes. La cystéine perd son pouvoir phagostimulant en absence de saccharose et ne semble donc pas avoir de propriétés propres à ce niveau. 


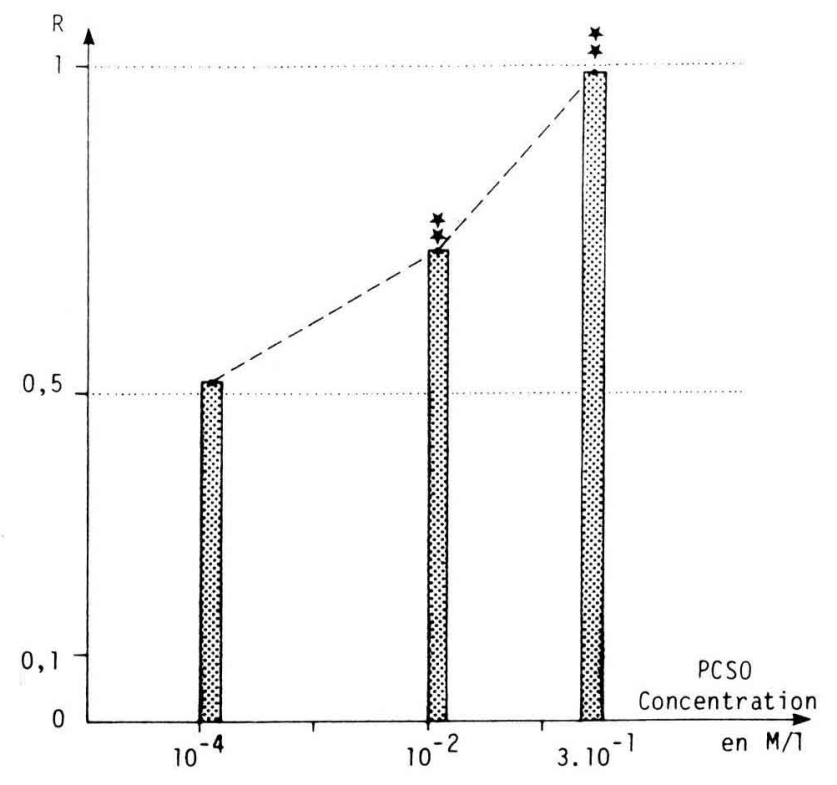

Fig. 3. Rapports $R$ des poids moyens consommés par les $L 5$ d'A. assectella en présence de diverses concentrations du précurseur soufré, le PCSO. Les 2 supports sont badigeonnés de saccharose. La présence d'astérisque indique que $E$ et $T$ sont significativement différents, soit $R \neq 0,5$ (test de Wilcoxon). ${ }^{*}: 0,05>P>0,01 ;{ }^{* *}: 0,01>P$.

\section{Discussion}

La phagostimulation chez les L5 d'A. assectella présente de nombreuses similitudes avec celle étudiée chez d'autres insectes.

Trois des sucres testés, le saccharose, le fructose et le glucose, sont des sucres libres trouvés dans les Allium (Bacon, 1959; Boscher, 1969), tandis que le galactose et le xylose ont été identifiés dans certains glycosides (Starke et Herrmann, 1976; Harmatha et al., 1987). Le saccharose est connu comme étant très stimulant, de même que, à un degré moindre, le glucose et le fructose (Bernays et Simpson, 1982; Ladd, 1986). Quant au galactose, il est généralement peu ou pas actif. Les résultats obtenus avec ces sucres chez $A$. assectella sont donc glo- balement en accord avec ceux obtenus par ailleurs. Cependant, plus intéressante est l'action du xylose qui est souvent répulsif (Chippendale et Reddy, 1974; Ladd, 1986) alors qu'il est plus phagostimulant pour $A$. assectella que le saccharose. II serait intéressant de connaître la quantité des pentoses contenus dans les Allium, alors que ceux-ci ont été peu étudiés et qu'ils sont habituellement peu ou pas utilisés par les insectes (House, 1974). Un tel résultat devra être confirmé ultérieurement.

Parmi les autres substances testées, l'acide ascorbique présente un effet phagostimulant. Or cette vitamine, abondante dans certains Allium (Rousseau, 1940; Abdou et al., 1972), est phagostimulante pour de nombreux insectes (Bernays et Simpson, 1982) et ceux-ci en ont fré- 


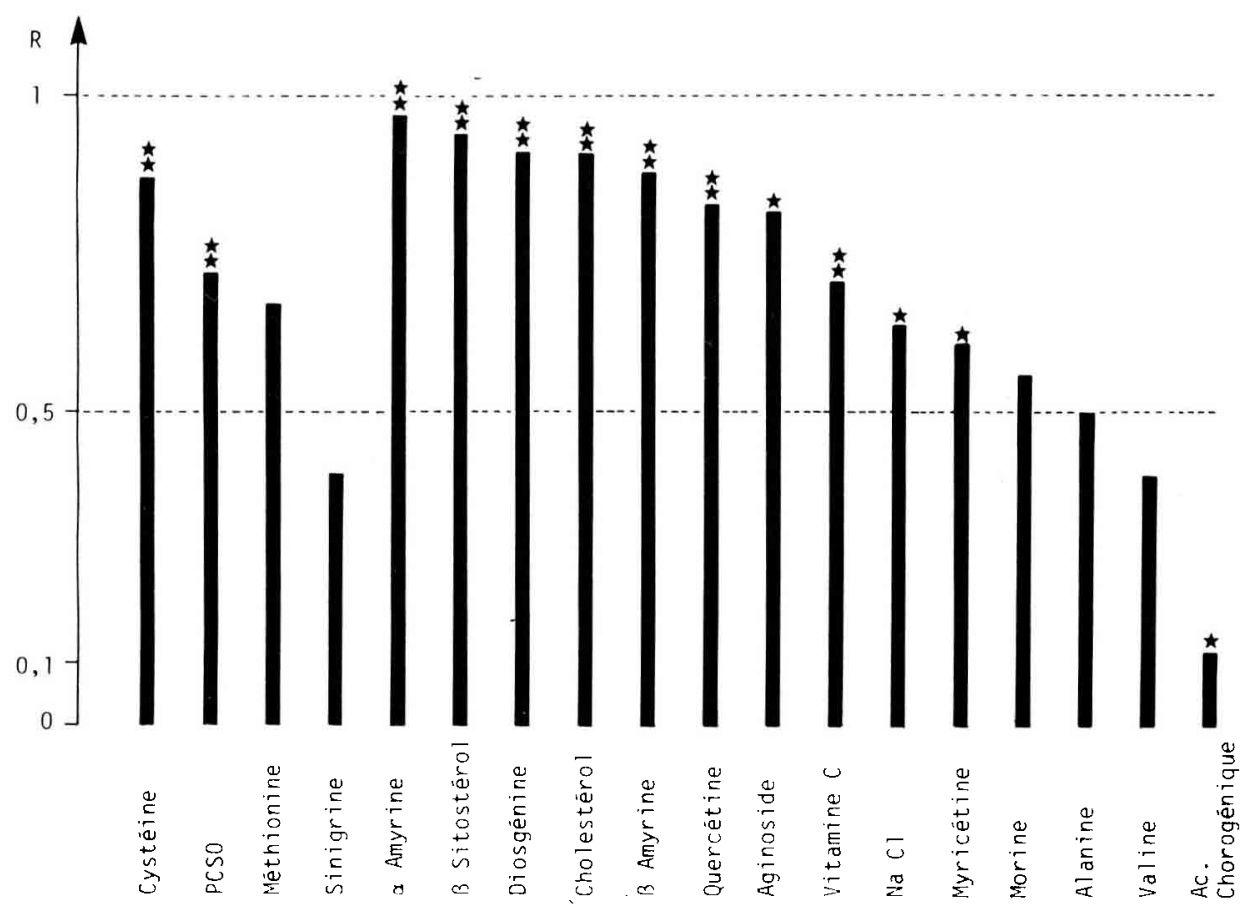

Fig. 4. Rapports $R$ des poids moyens consommés par les $L 5 d^{\prime} A$. assectella en présence de 18 substances non volatiles. Les 2 supports sont badigeonnés de saccharose. Les substances à poids moléculaire élevé (stéroïdes, saponines, flavonoïdes) sont testées à la concentration de $2.10^{-2} \mathrm{M} / 1$ et les autres substances à la concentration de $4.10^{-2} \mathrm{M} /$. La présence d'astérisque indique que $E$ et T sont significativement différents, soit $\mathrm{R} \neq 0,5$ (test de Wilcoxon). ${ }^{*}: 0,05>P>0,01{ }^{* *}: 0,01>P$.

quemment besoin dans leur alimentation (House, 1974).

Des 4 flavonoïdes testés, la quercétine, présente dans les Allium (Starke et Herrmann, 1976), stimule de façon importante l'alimentation des L5 d'A. assectella. II semble que ces composés soient souvent phagostimulants pour les insectes spécialistes des plantes les produisant. La morine du mûrier stimule Bombyx mori (Hamamura, 1970) et l'acide chlorogénique de la pomme de terre stimule le doryphore (Hsiao et Fraenkel, 1968). La reconnaissance sensorielle par ces insectes est donc très précise puisque les structures de ces divers flavonoïdes sont très semblables les unes aux autres.
Les différents stéroïdes testés sont tous phagostimulants. Ils ont été identifiés dans différents Allium : les amyrines en très faible quantité dans l'oignon (Smoczkiewicz et al., 1982), de même que le sitostérol plus abondant, le cholestérol (Ito et al., 1977) et la diosgénine (Ismailov et Aliev, 1974). La valeur nutritive de ces substances est bien connue chez les insectes, particulièrement le B-sitostérol et le cholestérol (House, 1974). Leur effet phagostimulant a été rarement étudié, bien que le B-sitostérol soit l'un des composés des feuilles de mûrier actif sur Bombyx mori (Hamamura, 1970).

L'aginoside, saponine stéroïdique, est faiblement phagostimulant mais égale- 


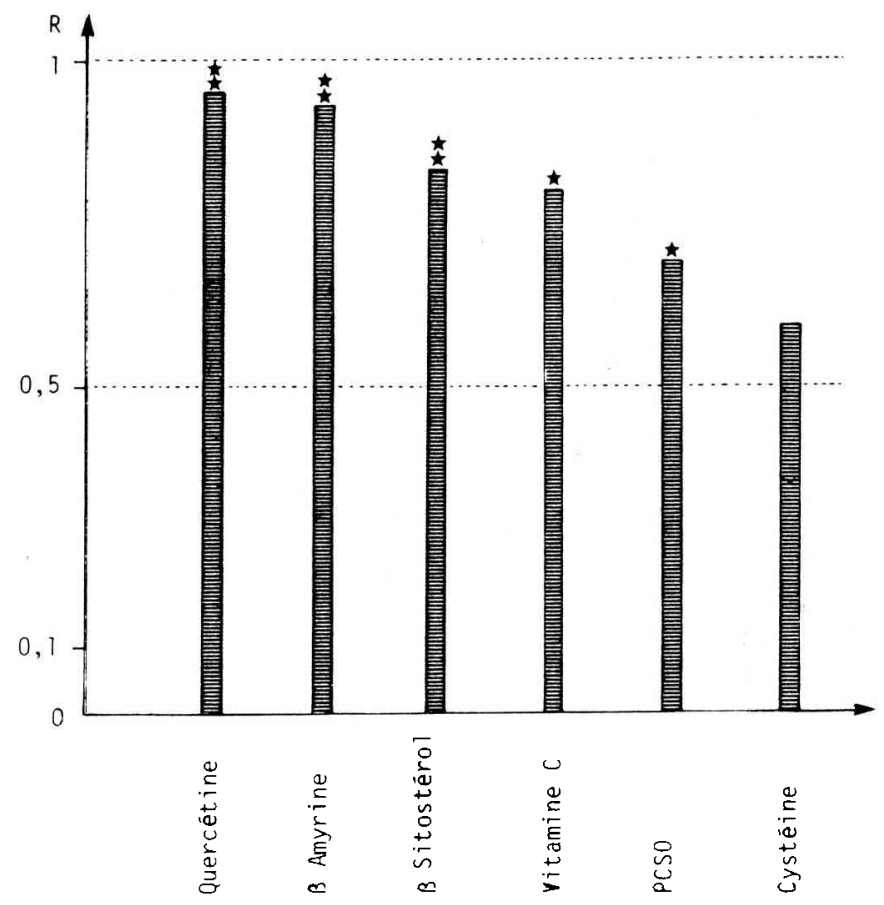

Fig. 5. Rapports des poids moyens consommés par les L5 d'A. assectella en présence de 6 substances non volatiles présentées seules. Les supports ne sont pas recouverts de saccharose. La présence d'astérisque indique que $E$ et $T$ sont significativement différents, soit $R \neq 0,5$ (test de Wilcoxon). ${ }^{*}: 0,05>P>0,01 ;{ }^{* *}: 0,01>P$.

ment toxique pour les larves d'A. assectella (Harmatha et al., 1987). Comment un tel mécanisme a-t-il pu évoluer ? On peut envisager que l'activité de cette saponine provienne du glycoside composé notamment de restes de glucose et xylose. Une autre possibilité est que l'aginoside étant essentiellement trouvé dans les fleurs, les larves qui consomment surtout les limbes ont été peu en contact avec cette substance au cours de l'évolution.

Le PCSO, substance spécifique des Allium, est phagostimulant. II intervient probablement dans la sélection des Allium par les larves d'A. assectella. Cela rappelle parfaitement l'action de la sinigrine, précurseur de l'isothiocyanate d'allyle, sur l'alimentation des insectes spécialistes des crucifères (Thorsteinson, 1953).

Des 4 acides aminés testés, seule la cystéine accroît la prise de nourriture des L5 d'A. assectella. Or, la méthionine est fréquemment un meilleur phagostimulant que la cystéine (Bernays et Simpson, 1982). De plus, la cystéine et la cystine sont rarement indispensables à la nutrition des insectes, contrairement à la méthionine (House, 1974). On pouvait donc s'attendre à une action importante de la méthionine. L'action de la cystéine montre que les $L 5$ ne sont pas sensibles à toutes les substances soufrées. Cela est confirmé d'autre part par l'expérience réalisée avec la sinigrine. La cystéine étant, 
en outre, un des acides aminés "précurseur" du PCSO, on peut considérer que ce composé fait partie des phagostimulants spécifiques des Allium.

Le chlorure de sodium montre une légère action phagostimulante chez $A$. assectella comme chez Pieris brassicae (Ma, 1972) alors que, chez Trichoplusia $n i$, seuls les ions potassium ont une action synergisante sur la phagostimulation (Gothilf et Beck, 1967).

Le support témoin en papier-filtre employé lors du test concernant les cires épicuticulaires étant de beaucoup le témoin le plus consommé par les L5, la cellulose pourrait être phagostimulante pour cet insecte comme cela semble être le cas pour Bombyx mori (Hamamura, 1970). L'absence de différence significative, en revanche, entre le support témoin et le support test $(R=0,54)$ indique clairement que les cires ne sont pas phagostimulantes, bien que la nature et la proportion des différents constituants soient assez spécifiques de chaque espèce végétale (Cassagne et Lessire, 1974). Elles sont cependant phagostimulantes, notamment pour certains acridiens (Chapman, 1977) et pour Manduca sexta (de Boer et Hanson, 1988). Mais ces espèces peuvent être qualifiées d'cexophytophages" constamment en contact avec les cires, tandis que les larves d' $A$. assectella sont des "endophytophages" qui vivent en mineurs dans le limbe de la plante-hôte sans contact avec les cires superficielles.

A l'exception des sucres, toutes les substances étudiées l'ont été en présence de saccharose à faible concentration. Les expériences ne permettent donc pas de distinguer un effet phagostimulant propre d'un effet synergique bien étudié chez Leptinotarsa decemlineata par exemple (Hsiao, 1972), espèce pour laquelle le saccharose ne devient phago- stimulant qu'en présence d'autres composés comme l'alanine ou la lécithine. Chez $A$. assectella, un effet synergique semble bien réel pour la cystéine qui n'est plus phagostimulante en absence de saccharose. Bien que tous les composés testés précédemment ne laient pas été à nouveau sans saccharose, cette synergie semble peu fréquente chez la teigne du poireau, puisque 5 des 6 composés testés seuls sont phagostimulants par euxmêmes.

Malgré l'absence de tanins et d'alcaloïdes dans le genre Allium (Rousseau, 1940), le choix de la nourriture chez $A$. assectella pourrait être influencé par des antiappétents. En effet, en présence d'acide chlorogénique, $R$ est significativement inférieur à $0,5(R=0,12)$. D'autre part, certains extraits de plantes n'appartenant pas au genre Allium sont antiappétents (Al-Rouz et Thibout, 1988a).

\section{Conclusions}

Le contrôle de la prise de nourriture chez les L5 d'A. assectella, comme chez la plupart des insectes, pourrait donc dépendre de 4 catégories de produits :- des composés stimulants assez généraux comme les sucres, la vitamine $\mathrm{C}$ ou certains stéroïdes; il s'agit souvent de composés intervenant dans les phénomènes de nutrition; - des allélochimiques peu abondants rencontrés dans les Allium et dans d'autres plantes, comme les amyrines; - des composés inhibiteurs généraux ou non qui doivent être peu abondants dans les plantes-hôtes d'A. assectella; enfin, d'autres composés stimulants spécifiques des Allium, plus ou moins dérivés de la cystéine. Les proportions relatives de ces 4 catégories de composés apportent au phytophage spé- 
cialiste un certain nombre d'informations gustatives nécessaires à la prise de nourriture et au choix de la plante-hôte.

Ces premières études sur la prise alimentaire des L5 doivent s'étendre aux autres stades, en particulier au $1^{\text {er }}$ stade néonate qui choisit le lieu de pénétration et se trouve donc, à un moment, en contact avec les cires épicuticulaires. De plus, des travaux réalisés à l'aide de milieux nutritifs synthétiques doivent permettre d'aborder l'influence du conditionnement sur les «habitudes" alimentaires des larves. Enfin, les substances soufrées volatiles peuvent également avoir un effet phagostimulant.

\section{Remerciements}

Les auteurs tiennent à remercier le Dr J. Harmatha de l'Académie des sciences de Prague qui leur a aimablement fourni l'aginoside et le Dr J. Auger de I'IBEAS de Tours qui a synthétisé le PCSO. Ils remercient également Mr J.C. Landré pour son aide technique et iconographique.

\section{Références}

Abdou I.A., Abou-Zeid A.A., El-Sherbeeny M.R. \& Abou-el-Ghet Z.H. (1972) Antimicrobial activities of Allium sativum, Allium cepa, Raphanus sativus, Capsicum frutescens, Eruca sativa, Allium kurrat on bacteria. Qual. Plant. Mater. Veg. 22, 29-35

Al-Rouz H. \& Thibout E. (1988a) Premières observations et rôle des Allium sur le comportement alimentaire des larves de la teigne du poireau, Acrolepiopsis assectella. Acta Ecol. Ecol. Applic. 9, 261-273

Al-Rouz H. \& Thibout E. (1988b) Analyse en olfactomètre de l'attraction des larves d'Acrolepiopsis assectella par des substances allélochimiques. Entomol. Exp. Appl. 47, 231-237

Bacon J.S.D. (1959) The trisaccharide fraction of some monocotyledons. Biochem. J. 73, 507514
Bernays E.A. \& Simpson S.J. (1982) Control of food intake. Adv. Insect. Physiol. 16, 59-118

Boer G. de \& Hanson F.E. (1988) The role of leaf lipids in food selection by larvae of the tobacco hornworm, Manduca sexta. J. Chem. Ecol. 14, 669-682

Boscher J. (1969) Formation, structure et germination des bulbilles inflorescentielles de l'ail des vignes (Allium vineale L.). Ann. Sci. Nat. Bot. 10, 375-468

Cassagne C. \& Lessire R. (1974) Etude de la biosynthèse des alcanes dans l'épiderme des feuilles d'Allium porrum L. II. Synthèse des acides gras et leur relation avec les alcanes. Physiol. Veg. 12, 149-163

Chapman R.F. (1977) The role of the leaf-surfa$c e$ in food selection by Acridids and other insects. Coll. Inter. CNRS Comportement des insectes et milieu trophique 265, 133-149

Chippendale G. \& Reddy G.P.V. (1974) Dietary carbohydrates: role in feeding behaviour and growth of the southwestern corn borer, Diatraea grandiosella. J. Insect. Physiol. 20, 751759

Gothilf S. \& Beck S.D. (1967) Larval feeding behaviour of the cabbage looper, Trichoplusia ni. J. Insect. Physiol. 13, 1039-1053

Hamamura Y. (1970) The substances that control the feeding behavior and the growth of the silkworm Bombyx mori L. : 55-80. In : Control of Insect Behavior by Natural Products (Wood D.L., Silverstein R.M. \& Nakajima M., eds.). Academic Press, New York

Harmatha J., Mauchamp B., Arnault C. \& Slama K. (1987) Identification of a spirostanetype saponin in the flowers of leek with inhibitory effect on growth of leek-moth larvae. Biochem. System. Ecol. 15, 113-116

House H.L. (1974) Nutrition : 1-62. In : The physiology of Insecta, 2nd ed. (Rockstein M., ed.), Academic Press, New York

Hsiao T.H. (1972) Chemical feeding requirements of oligophagous insects : 225-240. In : Insect and Mite Nutrition (Rodriguez J.G., ed.). North Holland, Amsterdam

Hsiao T.H. \& Fraenkel G. (1968) Isolation of phagostimulative substances from the host plant of the Colorado potato beetle. Ann. Entomol. Soc. Am. 61, 476-484

Ismailov A.I. \& Aliev A.M. (1974) Study of sapogenins from the white onion growing in Azerbaidzhan. Uch. Zap. Azerb. Gos. Med. Inst. 37, 60-64 
Ito T., Tamura T., Mitsuhashi T. \& Matsumoto T. (1977) Sterols in Lyliaceae. Phytochemistry 16, 140-141

Ladd T.L. Jr. (1986) Influence of sugars on the feeding response of Japanese beetles (Coleoptera : Scarabaeidae). J. Econ. Entomol. 79, 668-671

Lecomte C. \& Thibout E. (1981) Attraction d'Acrolepiopsis assectella en olfactomètre, par des substances allélochimiques volatiles d'A/lium porrum. Entomol. Exp. Appl. $30: 293-300$

Ma W.C. (1972) Dynamics of feeding responses in Pieris brassicae $L$. as a function of chemosensory inpout : A behavioural, ultrastructural and electrophysiological study. Meded. Landbouwhogesch. Wageningen 11, 162

Meisner J. \& Ascher R.S. (1968) A method to assay the phagostimulatory effect towards insects of plant extracts applied to styropor disks. Rev. Parasitol. 29, 74-77

Rousseau J. (1940) Recherches biochimiques sur le contenu vacuolaire des cellules des bulbes du genre Allium. Pub. Univ. Poitiers, Sci. Nat. 2, 6-114

Smoczkiewicz M.A., Nitschke D. \& Wieladek H. (1982) Microdetermination of steroid and triterpene saponin glycosides in various plant materials. I. Allium species. Mikrochim. Acta 2, 4353

Starke H. \& Herrmann K. (1976) Flavonole und Flavone der Gemüsearten. VII. Flavonole des Porrees, Schnittlauchs und Knoblauchs. $Z$. Lebensm. -Unters. -Forsch. 161, 25-30

Thorsteinson A.T. (1953) The chemotactic responses that determine host specificity in an oljgophagous insect (Plutella maculipennis (curt.) Lepidoptera). Can. J. Zool. 31, 52-72 